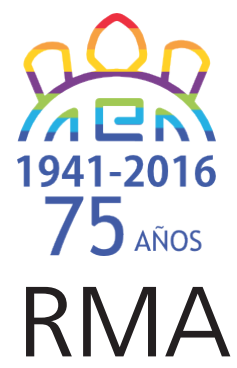

Arqueología

\title{
Una aproximación zooarqueológica a las prácticas de los cazadores-recolectores en el Alero Deodoro Roca (ca. 2970 AP), Valle de Ongamira, Córdoba.
}

\author{
A zooarchaeological approach to the practices of hunter- \\ gatherers in the Alero Deodoro Roca (ca. 2970 AP) \\ Ongamira Valley, Córdoba.
}

Thiago Costa*

*IDACOR-CONICET, Museo de Antropología, Facultad de Filosofía y Humanidades, Universidad Nacional de Córdoba. Av. H. Yrigoyen 174 (5000) Córdoba, Argentina. E-mail: thfcosta@gmail.com

\begin{abstract}
Resumen
El interés particular de este trabajo se centra en estudiar las relaciones entre los cazadores-recolectores y los animales, utilizando como caso de estudio los restos faunísticos recuperados en el sitio Alero Deodoro Roca (ADR), que según dataciones radiocarbónicas se ubican en el Holoceno tardío (ca.2970 AP). Para caracterizar dichas interacciones se plantea una metodología que involucra análisis faunísticos tafonómicamente orientados y de distribución espacial en el sitio, utilizando como herramienta principal los sistemas de información geográficos (SIG). De esta manera,se intenta integrar aspectos metodológicos de la arqueología interpretativa y de la arqueología científica. A partir de los resultados observados se interpreta una mayor presencia de ungulados en relación con otras especies, y dentro de estos se destacan los guanacos (Lama guanicoe). A la vez, los análisis tafonómicos sugieren una buena conservación del conjunto faunístico, aunque se han podido evidenciar alteraciones tanto naturales como antrópicas. Los resultados de los análisis espaciales indican la realización de actividades diversas de procesamiento y combustión, con una mayor concentración en el sector Oeste de las excavaciones. De acuerdo a lo anterior, se concluye que se desarrollaron actividades reiteradas de procesamiento con diferentes fines y consumo, especialmente de guanacos, en el sector B del sitio ADR.
\end{abstract}

Palabras clave: Zooarqueología; Cazadores-recolectores; Sierras Pampeanas; Córdoba.

\begin{abstract}
The paper focuses in studying hunter-gatherers and animals relations, using as a case study the faunal remains recovered in the Alero Deodoro Roca (ADR) site, which according to radiocarbon dating are located in the late Holocene (ca. 2970 AP). To characterize these interactions we propose a methodology that involves taphonomically oriented faunal analysis and spatial distribution on the site, using as main tool, geographic information systems (GIS). Thus, it attempts to integrate methodological aspects of interpretive archeology and scientific archeology. Based on our results, we suggest greater presence of ungulates in relation to other species and within these, guanacos (Lama guanicoe) are the majority. Also, taphonomic analyzes suggest good conservation of faunal assemblage, although there are evidence of both, natural and anthropogenic actions in the faunal remains. Distributional analysis results indicate various processing and combustion activities, although higher concentrations in the western sector of the excavations were noticed. According to the above, we concluded that repeated processing activities, with different purposes, and (especially guanaco) consumption was developed in the sector $B$ of ADR site.
\end{abstract}

Keywords: Zooarchaeology; Hunter-gatherers; Southern Pampean Hills; Córdoba.

El interés particular de este trabajo se centra en estudiar las relaciones que los grupos cazadores-recolectores mantuvieron con los animales, las cuales pueden ser más complejas que la mera apropiación de insumos para la subsistencia, ya que en general, estas sociedades no perciben el mundo natural radicalmente separado de los humanos (ver Fausto 2007; Hill 2011; Overton y Hamilakis 2013; entre otros). En este sentido, las interacciones humano-animal en estas sociedades comprenden una amplia gama de asociaciones (económicas, simbólicas, 
sociales, etc.) que van más allá de las proteínas y calorías (Russell 2012).

Tomando como ejemplo el caso de los grupos amerindios sudamericanos con ontología animista, parece interesante el abordaje de Fausto (2007). El autor toma datos de 53 pueblos pertenecientes a distintos grupos lingüísticos de la región, y propone que la predación manifestada en la cacería debe ser entendida como un deseo de producir una sociabilización interespecífica. De esta manera, demuestra la importancia que estos cazadores ponen en eliminar la agencia de las presas (que son vistas como iguales) a través de diferentes prácticas como, el correcto descuartizado de los animales, la cocción exagerada, o la evitación de consumir la propia caza o partes de la misma (ibíd. 2007). El caso manifestado por Fausto (2007) exhibe como aspectos ideológicos pueden manifestarse en las acciones que dejan huellas materiales, y por esta razón considerar estos aspectos ontológicos/ideológicos permitirá un acercamiento menos etnocéntrico y más complejo al registro arqueológico.

Bajo esta postura, el trabajo intenta integrar aspectos metodológicos de la arqueología interpretativa y de la arqueología científica (Hegmon 2003), a través de una serie de pasos que combinan los análisis tafonómicos y las aproximaciones analíticas interpretativas que podrían brindar información acerca de las prácticas de los grupos sociales en su relación con los animales no humanos (Marciniak 2001; Orton 2012). En este sentido, determinar los diversos procesos que alteraron el registro zooarqueológico cobra importancia, en términos de una tafonomía en positivo, ya que deberá contribuir a una comprensión más ajustada de las prácticas que vinculan los agentes humanos a los no-humanos (e.g. Lyman 1994).

De acuerdo a lo anterior, y con el objetivo de registrar información detallada acerca de las mencionadas interacciones, se ha elegido utilizar los Sistemas de Información Geográfica (SIG) para analizar los restos faunísticos recuperados en el sector B del sitio Alero Deodoro Roca (Valle de Ongamira, Córdoba) en diferentes escalas.

Con respecto a las plataformas SIG, actualmente existe una extensa bibliografía en donde se ha desarrollado y analizado con profundidad los aspectos teóricometodológicos de la arqueología relacionados con el uso de la herramienta (e.g. Maguire et al. 1991; Wheatley y Gillings 2002; entre otros). Desde sus principios, la extensión de sus aplicaciones fue sostenida por abarcar una variedad de temas como la prospección de superficie, el análisis espacial y territorial, o la gestión de inventarios de yacimientos (ver García Sanjuán 2003; Cattáneo et al.2015).

En el caso específico de la Argentina, la herramienta ha sido aplicada en la construcción de inventarios arqueológicos (Cattáneo et al. 2015); en el estudio de la tecnología lítica (Cattáneo et al. 2013a) y su distribución a nivel intrasitio (Sario y Salvatore 2013); en la elaboración de modelos predictivos (Matteucci y Scheinsohn 2004);en el análisis de patrones de asentamiento (Magnin 2013); de los paisajes de caza (Moreno 2013); del rol de paisajes culturales particulares (Gianotti y Bonomo 2013);entre varios otros (véase Figuerero Torres e Izeta 2013; Pastor et al. 2013).

Con una mirada proveniente de la zooarqueología, Izeta (2007, 2013) siguiendo la línea de Marean (Marean et al. 2001) y Abe (Abe et al. 2002), utiliza los SIG cambiando la escala en que se focalizaban los estudios. En lugar de investigar cuestiones territoriales, las unidades espaciales estudiadas pertenecían a los elementos óseos de los restos faunísticos investigados, y de esta forma, la herramienta termina siendo utilizada en la cuantificación de los elementos (MNE) o de las huellas antrópicas (Abe et al. 2002; Aguilar et al. 2011; Izeta 2013; Marean et al. 2001).

Por otro lado, la utilización de los SIG bajo el objetivo de analizar la dispersión de los restos faunísticos de un sitio, contribuye en las interpretaciones ya que brinda información significativa sobre la organización del asentamiento y los procesos pos-depositacionales sufridos por el mismo (Mainland et al. 2014; Marín-Arroyo 2008). En este sentido, ya son varios los trabajos que incluyen las herramientas SIG para estudiar la distribución espacial de los restos arqueofaunísticos (véase López et al. 2016; Mainland et al. 2014; Marín-Arroyo 2008). Por estas razones, los sistemas de información geográficos terminan siendo un importante elemento de contraste que auxilia en las interpretaciones del registro arqueofaunístico de diferentes maneras.

De acuerdo a lo previo, en este trabajo se presentarán análisis zooarqueológicos, tafonómicos y cuantitativos, asociados a la información de la distribución espacial a nivel intrasitio en el sector $B$ del Alero Deodoro Roca (ADR). Finalmente, el objetivo de los diferentes métodos es comprender la variación de las prácticas que vinculan humanos y animales en diferentes momentos ocupacionales del sitio durante el Holoceno tardío (ca. 2970 AP).

\section{Área de estudio}

El valle de Ongamira (Departamento de Ischilín, Córdoba) se encuentra en las Sierras Pampeanas Australes, sobre el extremo Norte de la unidad orográfica Sierras Chicas, casi al límite con las Sierras del Norte (Carignano 1999). El paisaje del valle se desarrolla sobre un conglomerado del basamento cristalino de Gondwana, que incluye una formación cretácica (arenisca roja) donde se forman paredones y aleros que presentan evidencias de ocupación humana (Izeta et al. 2014). El sitio conocido como Alero Deodoro Roca (ADR) es uno de estos abrigos, producto 


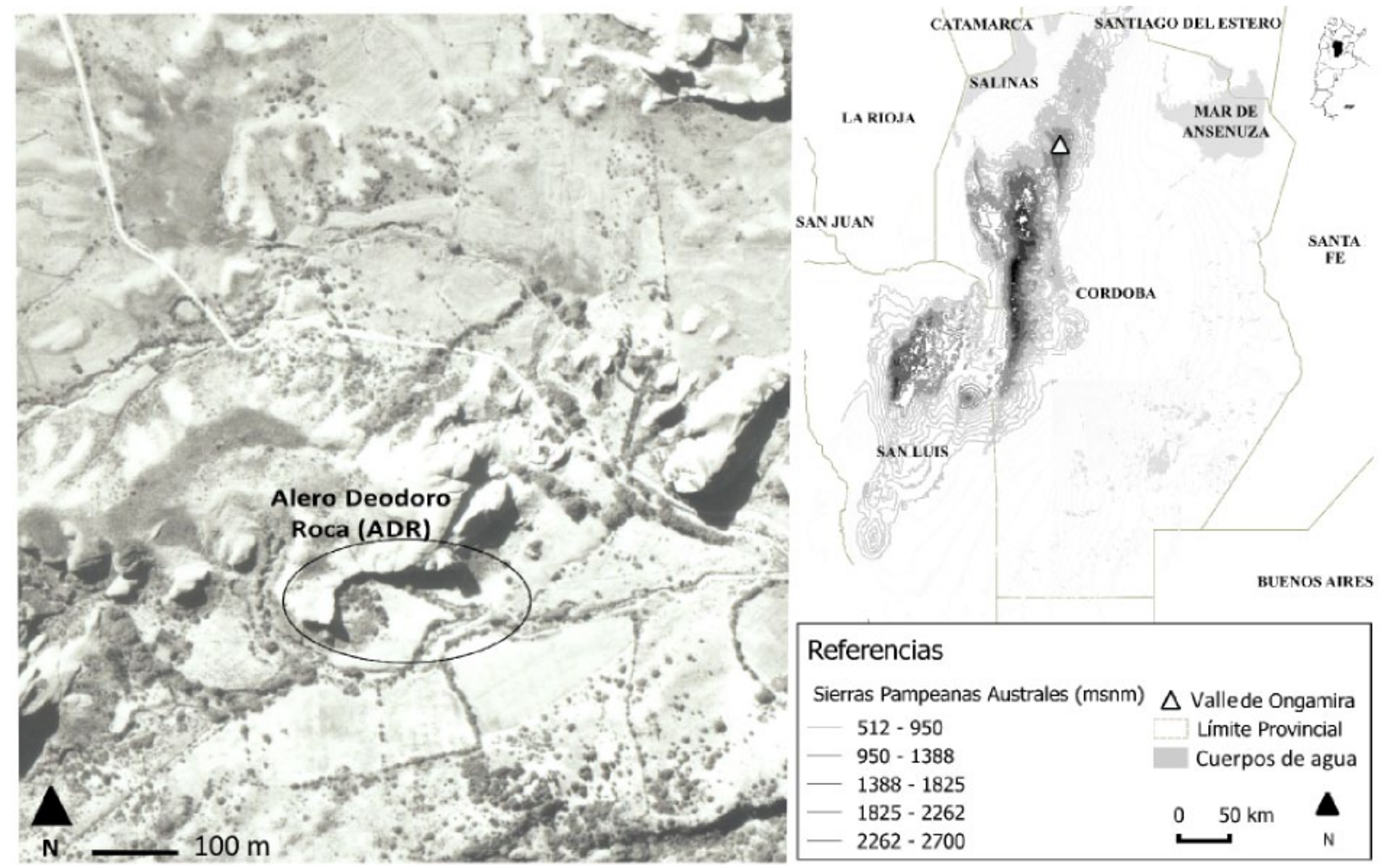

Figura 1. Mapa ubicando el Valle de Ongamira en la provincia de Córdoba (der.). Fotografía aérea localizando el sitio arqueológico ADR (izq.).

Figure 1. Map locating Ongamira Valley in the province of Cordoba (right). Aerial Photography localizing the archaeological site ADR (left).

de la erosión sobre las areniscas y conglomerados del sistema Gondwana (Cattáneo et al. 2013b; Costa 2015, 2016; Menghin y González 1954).

En términos eco-regionales, el área de estudio pertenece al Gran Chaco, uno de los mayores ecosistemas de Sudamérica y una de las florestas de ambientes secos más extensa del mundo (Zak et al. 2008). La eco-región se extiende por las planicies del cuaternario desde el Norte de Argentina, Oeste de Paraguay, pasando por el Sureste de Bolivia hasta el estado de Mato Grosso do Sul en Brasil (Zak et al. 2004). Desde la colonización española el Chaco es afectado por un proceso de intensa deforestación y sobrepastoreo que acarreó una invasión arbustiva, casi eliminando los pastizales abiertos (Leynaud y Bucher 2005).

Los cambios en el paisaje producidos por la colonización española, también afectaron a la distribución de las especies animales, simplificando hábitats y empobreciendo la biodiversidad (Cebollada Putz et al. 2012). De acuerdo a lo anterior, en términos zoogeográficos, el área de estudio fue clasificada por Ringuelet (1961) en la subregión Andino-patagónica de Dominio Central o Subandina. Hasta tiempos recientes eran abundantes las especies de gran porte, como Lama guanicoe, Hippocamelus sp. y Ozotocerus bezoarticus. En la actualidad hay registros de grandes aves, como Rhea sp., y la de artiodáctilos de menor tamaño, como Mazama guazoubira y Pecari tajacu. Asimismo, se reconoce la presencia de poblaciones aisladas de guanacos (Lama guanicoe) en el sector noroeste de la provincia, más específicamente en la región del Corredor Biogeográfico del Chaco Árido (Geisa 2012).

Las condiciones fisiográficas del valle se encuentran modeladas por un clima continental templado. El valle también se caracteriza por una mayor humedad relativa en comparación a las áreas circundantes y por la caída de nevadas excepcionales (Pastore y Methol 1953).

Asimismo,en el marco de un proyecto de investigación arqueológica en desarrollo (Cattáneo e Izeta 2016), Yanes y colaboradores (2014) recopeliaron datos paleoclimáticos para el Holoceno tardío (4500-1700 AP) a través de la caracterización isotópica del valle, utilizando muestras de agua de lluvia $\left(\delta^{18} \mathrm{O}\right)$, plantas actuales y materia orgánica del suelo $\left(\delta^{13} \mathrm{C}\right)$, carbonatos del sedimento en donde valvas de gasterópodos fueron preservadas $\left(\delta^{18} \mathrm{O}\right.$ y $\delta^{13} \mathrm{C}$ ) y valvas enteras de Plagiodontes sp. modernos y arqueológicos $\left(\delta^{18} \mathrm{O}\right.$ y $\delta^{13} \mathrm{C}$ ). Los resultados obtenidos por los autores demuestran valores de $\delta^{13} \mathrm{C}$ en media 2.5\%o más altos para las valvas arqueológicas respecto de las modernas. Estos valores estarían sugiriendo que durante el Holoceno tardío las plantas $\mathrm{C}_{4}$ fueron la principal fuente de alimentación de los Plagiodontes sp., mientras que en los especímenes actuales la alimentación se basaría en plantas $C_{3}$ (Yanes et al. 2014).

Los resultados arribados por los autores concuerdan 

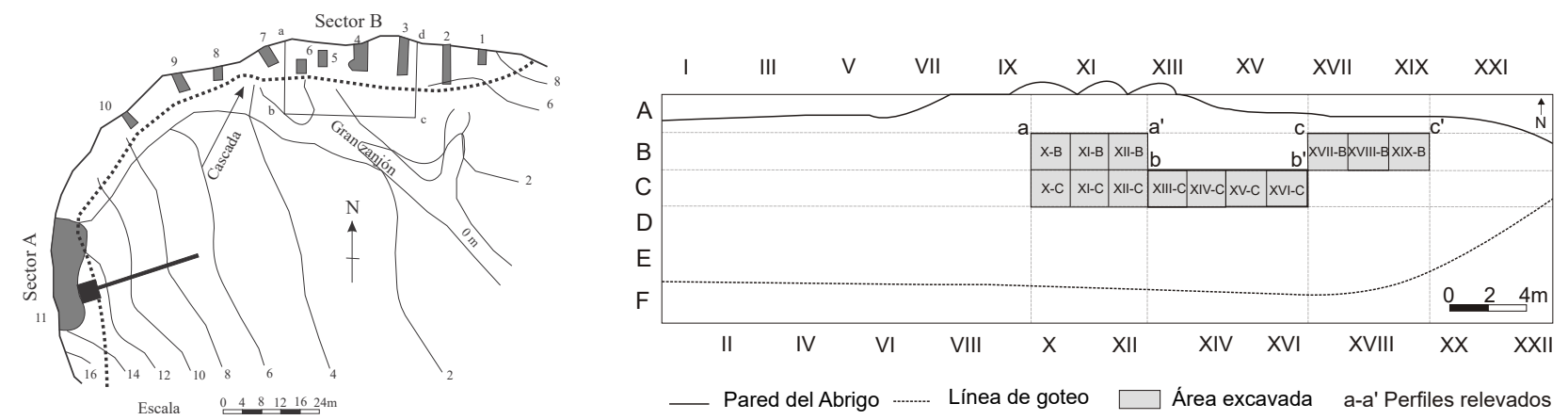

Figura 2. Alero Deodoro Roca (ADR) mostrando los sectores A y B. Detalle de los espacios intervenidos y perfiles relevados en el sector B del sitio ADR. Las cuadrículas en gris muestran las zonas del sector (B) en que se está trabajando en la actualidad.

Figure 2. Alero Deodoro Roca (ADR) showing the sectors $A$ and $B$. Details of the intervened spaces and registered profiles in the sector $B$ from $A D R^{\prime} s$ site. Gray areas show the places in sector (B) that are currently under study.

con otros obtenidos para el mismo periodo a través de diferentes proxies (ver Carignano 1999; Silva et al. 2011). En conjunto estarían apuntando a condiciones climáticas más secas y cálidas, con menor humedad relativa del aire, estaciones de lluvia marcadas y una predominancia de plantas $\mathrm{C}_{4}$ en la región. Alrededor de los 3500 AP el clima cambiaría gradualmente hacia las condiciones actuales; más frías, húmedas y con el predominio de las especies vegetales $C_{3}$ (Silva et al. 2011; Yanes et al. 2014). Por último, se resalta que este tipo de información contribuye en la comprensión de la ecología local y, por ende, en las interpretaciones del registro faunístico recuperado en el Alero Deodoro Roca (ADR).

\section{Investigaciones arqueológicas en el sitio ADR}

Los primeros acercamientos a la exploración del potencial arqueológico del ADR se dieron en la década de 1930, cuando Aníbal Montes llevó a cabo excavaciones en la localidad definiendo dos sectores para el alero, A y B (Cattáneo et al. 2013b; Montes 1943).De esta manera, el autor plantea 12 zanjas exploratorias, aunque centra sus esfuerzos en el sector A debido al potencial arqueológico y "la mejor conservación de los restos" (Montes 1943:231). Allí pudo determinar "cuatro pisos" de ocupación, pero sólo para el primero menciona la presencia de "trozos aislados de alfarería" (Montes 1943:238).

En el mismo volumen, Alberto Rex González (1943:143) realiza una descripción de lo que denomino "las piezas más demostrativas y características de todo el material encontrado". En lo que concierne a los restos faunísticos, el autor relata que pudieron recuperar gran cantidad de huesos fragmentados, en su mayoría de manera longitudinal, resaltando que algunos mostraban huellas de termoalteración. Asimismo en el piso 1 destaca la presencia de "cáscaras de huevos de avestruz y una enorme cantidad de conchas de Odontostomus", que supuso fueron utilizadas en la alimentación de las personas (González 1943:144).

Posteriormente, Osvaldo Menghin y Alberto Rex González vuelven a excavar el sitio trazando un cuadriculado en el sector B de ADR que subdividiría la superficie en transectas de $2 \mathrm{~m}$ cada una y con dirección Norte-Sur (Menghin y González 1954, véase sector B en Figura 2). De esta manera, estos autores pudieron recuperar las diferencias en la utilización del espacio intrasitio y la secuencia de ocupación.

Asimismo, Menghin y González (1954) vuelven a definir cuatro horizontes en sus investigaciones, llegando a la conclusión de que el III y IV reflejarían "culturas cazadoras-recolectoras". La aseveración resulta, por un lado, de las grandes cantidades de restos óseos procedentes de animales cazados, en particular restos de guanacos y ciervos. También es característica la presencia de gasterópodos y de fragmentos de huevos de ñandú, "...alimentos sumamente apetecido por los ocupantes..." del denominado horizonte III (Menghin y González 1954:234).

Es importante destacar que la caracterización de los restos faunísticos recuperados por los mencionados investigadores estuvo a cargo del paleontólogo Rosendo Pascual (1954). Según este autor, no se encontraron animales extintos, aunque tampoco se recuperó fauna europea. Los restos recuperados pertenecían "...en su totalidad a animales vivientes, característicos de la fauna neotropical" (Pascual 1954:269).

\section{Metodología}

Como ha sido mencionado previamente, el material analizado en el presente trabajo proviene de excavaciones estratigráficas de un proyecto en desarrollo actualmente. De esta forma, en todo el volumen excavado (aprox. 65 $\mathrm{m}^{3}$ ) fue posible identificar más de 114 rasgos y unidades estratigráficas, en contraposición a los cuatro Horizontes identificados por Montes (1943) y Menghin y González (1954). Estas diferencias demuestran "la complejidad de la composición del sitio y la simplificación realizada en la interpretación de la cronoestratigrafía del Sector B" (Cattáneo et al. 2013b:563).

Con el objetivo de solucionar la problemática planteada, 
durante las excavaciones del año 2010 se relevaron tridimensionalmente las posiciones de los restos óseos $(\geq 5$ $\mathrm{cm}$ ) en las diferentes unidades estratigráficas identificadas. Estas ubicaciones fueron integradas en una base de datos digital junto con variables obtenidas del análisis tafonómico, taxonómico y zooarqueológico general (véase Costa 2015). La información espacial agregada permitió generar mapas en dos (2D) y tres dimensiones (3D) que modelan la distribución de los hallazgos en tres cuadrículas, XIV-C, XV-C y XVI-C. Se optó por representar las mencionadas unidades porque comprenden el 73\% del conjunto óseo recuperado. Además han presentado un bajo grado de perturbaciones postdepositacionales percibidas durante los trabajos de campo (Cattáneo e Izeta 2016; Costa 2015, 2016).

Por otro lado, se destaca que la información recuperada en las cuadrículas aludidas anteriormente, y que serán tratadas en el presente trabajo, remite a diferentes momentos ocupacionales del Holoceno tardío ( 2970 AP). Es decir, son cuatro unidades estratigráficas datadas por AMS (de un total de 17 que presentaron restos de fauna con penecontemporaneidad, ver tabla 1), que exhibieron una fecha promediada en 2970 AP (Cattáneo et al. 2013b; Cattáneo e Izeta 2016; Costa 2015).

Para el desarrollo de la metodología se utilizó el software libre QGIS versión 2.14.2-Essen, con el complemento Qgis2threejs, que permite la visualización en tres dimensiones del mapa generado. Como se pretende demostrar adelante, la sistematización en el programa ha sido utilizada para ayudar en la compresión de la variación espacial, permitiendo reconocer patrones a través de las imágenes creadas y resaltando las características de los elementos analizados. Estos factores deberán contribuir a las inferencias acerca de las prácticas humanas en el pasado y los procesos de formación del Sector B del sitio ADR.

Con respecto a los aspectos metodológicos que guiaron la investigación zooarqueológica, se ha procedido a analizar el conjunto faunístico considerando; la identificación anatómica y taxonómica, las clases de edad (e.g. fusión epifisiaria y erupción dentaria, Mengoni Goñalons 1999; Kaufmann 2009), las unidades de cuantificación (NISP, Grayson 1984) y el análisis en las frecuencias de las porciones esqueletarias (MNE, Marean et al. 2001, y MAU, Binford 1978). Para el estudio de la anatomía económica se utilizó el Índice de Utilidad propuesto por Borrero (1990a). Asimismo se resalta la adopción de un enfoque tafonómico orientado a reconocer la historia de cada elemento recuperado (Borrero 1990b) y que busca identificar los agentes responsables por cada modificación involucrando tanto los procesos naturales como culturales (Gutiérrez 2009; Lyman 1994).

\section{Resultados}

\section{Los restos faunísticos recuperados}

De un modo general el conjunto arqueofaunístico del Sector B está constituido por taxones vivientes de la fauna neotropical, como ya se había advertido anteriormente (Pascual 1954, Costa 2015). Fue posible identificar elementos pertenecientes a taxones de diferentes tamaños corporales, es decir, pequeños (gasterópodos, aves, roedores, dasipódidos y carnívoros), medianos (reídos y cérvidos) y grandes (camélidos). Sin embargo, al observar la Tabla 2, es notable la mayor presencia de los ungulados (53\%) y dentro de este grupo, los animales de mayor talla (Ungulata grande y Lama guanicoe) representan el $48 \%$ del conjunto analizado.

Cabe destacar que los diversos fragmentos de valvas de moluscos fueron recuperados en forma de muestreo y tratados en otro trabajo (véase Izeta et al. 2014). De esta manera fue posible identificar grandes cantidades de gasterópodos Plagiodontes sp. asociados a las estructuras de combustión (Gordillo et al. 2013; Izeta et al. 2014). Finalmente, y con relación a los roedores, los análisis efectuados hasta el momento indican que su presencia es debido a la acción de aves rapaces strigiformes (Mignino et al.2016).

En cuanto al estado de los restos, la meteorización no ha afectado de manera significativa al conjunto. Apenas el $3,25 \%$ de los especímenes registrados han exhibido lo que se podría categorizar como meteorización alta $(\geq 4$ sensu Todd 1987). De igual forma el mordisqueo no ha sido un factor de alteración importante del registro, ya que solamente 41 especímenes exhibieron huellas con este tipo de alteraciones. Asimismo, el conjunto general manifiesta una alta fragmentación, con los fragmentos promediando los 33,33 mm de longitud (los ungulados presentan valores ligeramente más altos). Además, el $59 \%$ de los especímenes identificados ha mostrado señales de alteración térmica, aunque la mayoría de estos (48\%) exhibió baja termoalteración, es decir, mostraron coloración en tonalidades de marrón asociadas a una exposición al calor por cortos períodos (sensu Nicholson 1993).

\begin{tabular}{cccc}
\hline Unidad o rasgo fechado & Código de laboratorio & Fechado & Material fechado \\
\hline 50 & YU2293 & $2942+/-25$ & Carbón \\
7 & YU2291 & $2944+/-44$ & Carbón \\
34 & YU2290 & $2952+/-21$ & Carbón \\
65 & MTC14144 & $3043+/-41$ & Carbón \\
\hline
\end{tabular}

Tabla 1. Unidades estratigráficas datadas por radiocarbono y tratadas aquí. Corresponden al sector B del sitio Alero Deodoro Roca (ADR). Adaptado de Cattáneo et al. 2013b.

Table 1. Radiocarbon dated stratigraphic units treated here. Correspond to the sector $B$ of the Alero Deodoro Roca (ADR) site. Adapted from Cattaneo et al. $2013 b$. 
Por otro lado, el 5\% del conjunto exhibió depositación de $\mathrm{CaCO}_{3}$ y el $1 \%$ ha exhibido marcas ocasionadas por las acciones de las raíces de plantas. En cuanto a la depositación química mencionada, el $65 \%$ de los elementos afectados pertenecen al grupo de ungulados. Cabe destacar que la modificación natural parece estar relacionada a la presencia de moluscos, ya que se notan valvas y espículas de carbones asociados a la depositación del carbonato de calcio en los restos óseos (Costa 2015).

Con respecto a las marcas de procesamiento antrópico, las mismas fueron identificadas en el $11 \%$ del conjunto y de estos el $86 \%$ son ungulados. Si se observa el subconjunto de guanacos, como ejemplo, se percibe que ambos grupos etarios (adultos y subadultos) han sido sometidos a acciones de procesamiento (Figura 3). En este sentido, fue posible identificar huellas de cortes y raspados tanto en especímenes relacionados a individuos subadultos (NISP=9) como en adultos (NISP=21); los negativos de impactos también se observaron en los animales inmaduros (NISP=21), así como en los maduros (NISP=62). Acorde a estas marcas, es notable el grado de fragmentación del conjunto, que ha mostrado una longitud promedio de 53,66 mm, cuando se consideran únicamente los fragmentos con fracturas frescas.

En cuanto al perfil anatómico expresado mediante el $\% \mathrm{MAU}$, las muestras asignadas a guanacos adultos exhiben frecuencias bajas (valor promedio de $27 \%$ ) para los elementos apendiculares delanteros y medianas para los apéndices traseros (35\%). Con relación a los animales inmaduros, los resultados del \%MAU han brindado frecuencias promediadas altas para los elementos apendiculares delanteros (52\%) y bajas para los traseros (26\%). A la vez, los elementos pertenecientes al esqueleto axial presentan frecuencias bajas, un promedio de $8 \%$ en ambos casos, animales subadultos y adultos.

Asimismo, es llamativa la ausencia de algunos elementos en ambos grupos (e.g. escápula, Figura 3). Los mencionados resultados podrían estar indicando una utilización diferenciada del esqueleto apendicular para animales adultos y subadultos, o quizás que las actividades variaban en las diferentes ocupaciones del sector B de ADR, durante el periodo en cuestión.

Por otro lado, se resalta la mayor representación de elementos relacionados al esqueleto apendicular (64\%) en los demás grupos de ungulados recuperados. En este sentido, se supone que estos elementos estén más fragmentados (y por esta razón más representados) como consecuencia del procesamiento antrópico (para Lama guanicoe véase las representaciones de impactos en la Figura 3, para el restante de los ungulados ver Costa 2015).

De esta manera, al correlacionar los valores del MAU\% y del MUI\%, las curvas obtenidas (Figura 4) apuntaron a una práctica relacionada al procesamiento inicial de las carcasas (sensu Binford 1978). No obstante, los resultados de las correlaciones fueron negativos y poco significativos tanto para los guanacos adultos ( $r s=-0,06$ y $p=0,69$ ) como para los subadultos ( $r s=-0,05$ y $p=0,77)$. Los altos rangos de error obtenidos no permiten sugerir que hubo una práctica de selectividad basada en el aporte cárnico de las unidades anatómicas.

Asimismo, es notable que en ambos grupos etarios

\begin{tabular}{|c|c|c|c|c|c|c|c|c|c|c|c|c|c|c|c|c|c|c|}
\hline \multirow[t]{2}{*}{ Taxón } & \multicolumn{17}{|c|}{ NISP } & \multirow[t]{2}{*}{ Total } \\
\hline & 6 & 7 & 10 & 11 & 14 & 22 & 34 & 35 & 45 & 50 & 52 & 59 & 60 & 61 & 62 & 63 & 65 & \\
\hline Gastropoda & 13 & 69 & - & - & - & 2 & - & 1 & - & - & - & 11 & 53 & 14 & - & - & - & 163 \\
\hline Aves & 3 & 1 & - & - & 1 & - & - & - & - & - & - & 1 & 1 & - & - & - & - & 7 \\
\hline Rhea americana & 2 & 20 & - & - & - & - & - & - & 1 & - & - & - & 3 & - & - & - & - & 26 \\
\hline Mammalia & 356 & 635 & 8 & 4 & 166 & 7 & 166 & 22 & 1 & 56 & 59 & 94 & 232 & - & - & - & - & 1806 \\
\hline Rodentia & 27 & 85 & - & 13 & 7 & 2 & 5 & 3 & 4 & 2 & 4 & 11 & 27 & 26 & - & - & - & 216 \\
\hline Dasypodidae & - & 4 & - & - & - & - & 2 & - & - & - & 2 & 1 & 1 & - & - & - & - & 10 \\
\hline Carnivora & - & - & - & - & - & - & - & - & - & - & - & 3 & 1 & - & - & - & - & 4 \\
\hline Ungulata medianos & 31 & 135 & - & 2 & 26 & - & 10 & - & - & - & 7 & 10 & 16 & - & 1 & - & - & 238 \\
\hline Mazama guazoubira & 3 & 16 & - & - & - & - & 2 & - & - & - & - & - & - & - & 5 & - & - & 26 \\
\hline Ozotocerus bezoarticus & - & 4 & - & - & 1 & - & - & - & - & - & - & - & - & - & - & - & - & 5 \\
\hline Ungulata grandes & 226 & 668 & 6 & 27 & 123 & 14 & 25 & - & 16 & 23 & 14 & 26 & 58 & 19 & - & - & - & 1245 \\
\hline Lama guanicoe & 164 & 441 & 10 & 5 & 128 & 4 & 35 & 5 & 7 & 58 & 7 & 92 & 113 & 12 & - & 6 & 4 & 1091 \\
\hline Total & 825 & 2078 & 24 & 51 & 452 & 29 & 245 & 31 & 29 & 139 & 93 & 249 & 505 & 71 & 6 & 6 & 4 & 4837 \\
\hline
\end{tabular}

Tabla 2. Taxones identificados en el sitio ADR, separados por las unidades estratigráficas que componen el bloque fechado en 3000 AP.

Table 2. Identified taxa in ADR site, separated by stratigraphic units from the 3000 BP block. 
algunos elementos de mayor rendimiento (e.g. fémur) estén presentes, aunque en baja frecuencia, lo que sugiere que las prácticas de las personas variaron en los diferentes momentos de ocupación del sitio. A la vez, los elementos faltantes podrían haber sido descartados en otros sectores aún no excavados (quizás de manera diferenciada), o haber sido formatizados y transportados a otros sitios.

De acuerdo a lo previo, el registro del instrumental lítico presenta una alta variabilidad tipológica, con al menos 15 clases de instrumentos detectados hasta el momento (Caminoa 2016). Además, gran parte de estos objetos estarían asociados a la formatización de artefactos en materias primas blandas (e.g. raspadores, raclettes, muescas, cuñas, entre otros). Aunque también se identificaron elementos que podrían estar siendo utilizados en el procesamiento relacionado al consumo de un animal (e.g. cortantes, cuchillos y raederas). Asimismo, los análisis sobre los grupos no-tipológicos apuntan a la ocurrencia de prácticas relacionadas a la "producción, uso, mantenimiento y descarte" de los instrumentos líticos en el sitio (Caminoa 2016:84). La sumatoria de estas evidencias sugiere que las prácticas debieron haber variado en los diferentes eventos de ocupación de ADR.

\section{Distribución de la fauna en ADR (ca. 2970 AP)}

Es notable una mayor representación de los fragmentos óseos ( $\mathrm{N}=412)$ en el sector oeste de las excavaciones (Figura 5A cuadricula XIV-C y porción colindante de la XV$C)$, hecho que parece apuntar a una concentración más intensa de las actividades en el área. A la vez, la distribución horizontal de los especímenes (Figura 5B) también parece indicar que ocurrió una mayor cantidad de eventos en la zona, donde fueron identificadas diferentes estructuras de combustión que parecen haber sido utilizadas por cortos periodos de tiempo (UE's 22-65, véase Cattáneo e Izeta 2016; Robledo 2016). Por otro lado, en el sector Este de la excavación (cuadricula XVI-C en la Figura 5) los restos faunísticos se encontraban casi en su totalidad, en la matriz estratigráfica (UE 7). Se identificaron también algunas áreas de combustión y un fogón, aunque en menores concentraciones, en las cuadriculas XV-C y XVI-C (para más ver Cattáneo e Izeta 2016; Robledo 2016). Además parece interesante destacar la ausencia de restos recuperados con medidas tridimensionales en el sector Centro-Norte de la excavación (XV-C), que corresponde a un fogón denominado UE 45 (NISP=29, véase Tabla 2).

En este sentido, los detalles exhibidos en la Figura $5 \mathrm{C}$ demuestran que los restos óseos sufrieron mayores daños en el sector oeste de las excavaciones (XIV-C y porción colindante de la XV-C). En lo que se refiere a la distribución
Los restos óseos con información tridimensional pudieron ser recuperados en 12 de las unidades estratigráficas asociadas al bloque temporal 2970 AP. De esta manera, fue posible analizar espacialmente 595especímenes, de los cuales 469 pudieron ser identificados taxonómicamente. Asimismo, es importante destacar que el $71 \%$ de los restos recuperados y representados en los mapas, pertenecen al grupo de artiodáctilos (Ungulados grandes, Lama guanicoe, Mazama guazoubira). Igualmente, se recuperaron con datos tridimensionales algunos gasterópodos (Plagiodontes sp.) y roedores (Ctenomys sp.) que se encontraban asociados a los primeros.

Figura 3. Esquemas del esqueleto de guanaco (Lama guanicoe) presentando MAU\% para individuos adultos (der.) y subadultos (izq.), y abajo las posiciones de las huellas de procesamiento antrópico identificadas en el ungulado.

Figure 3. Guanaco (Lama guanicoe) skeleton schemes presenting MAU\% for adult individuals (right) and subadults (left). The identified anthropic processing traces positions in the ungulate are shown below.
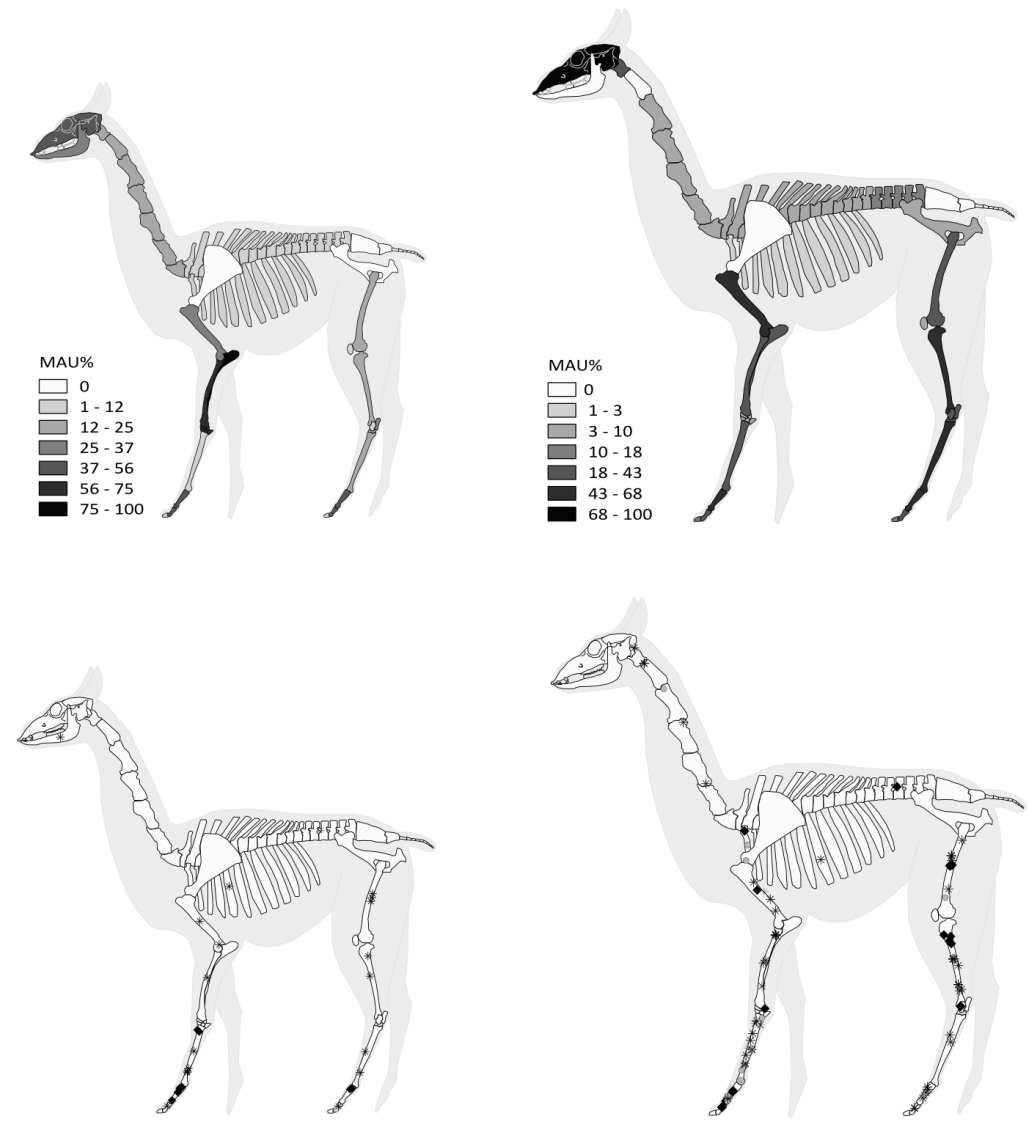

Guanacos subadultos

Guanacos adultos

Marcas antrópicas [31]

- Cortes y raspados [9]

* Impactos [21]

- Fracturados por procesamiento (posibles cortes e impactos) [1] 


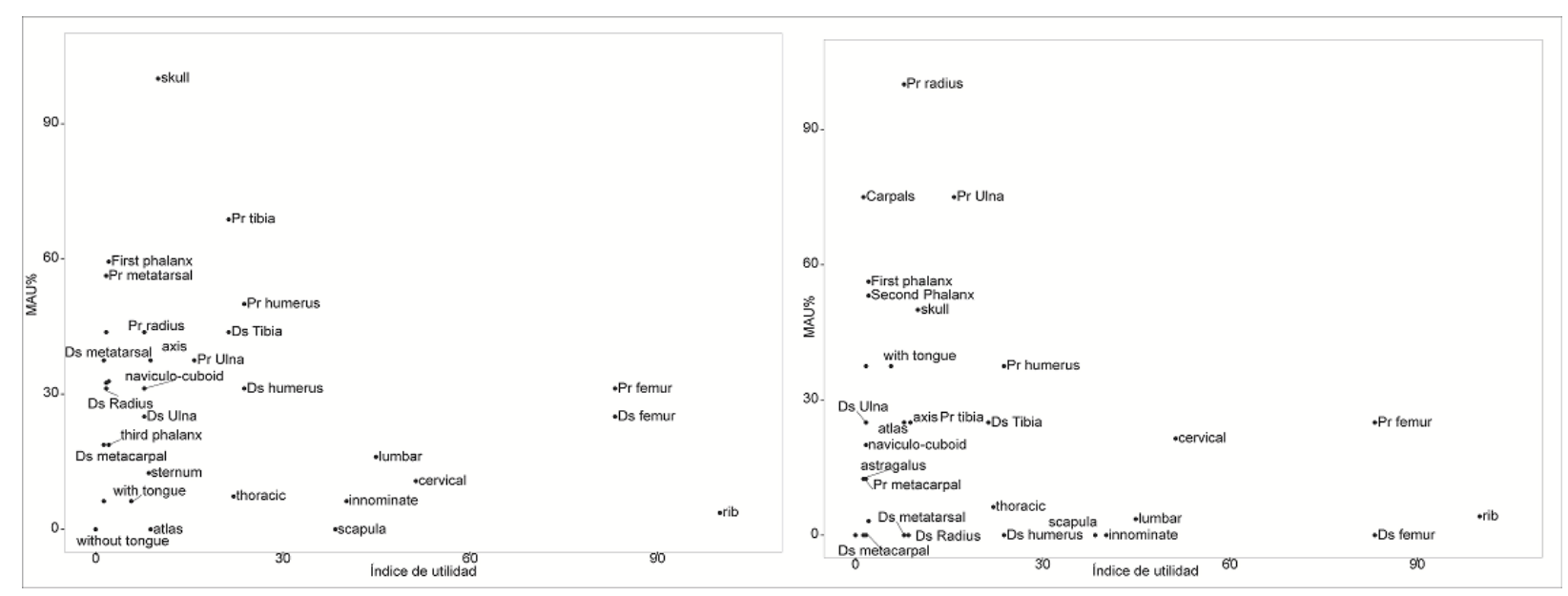

Figura 4. Gráficos de dispersión del Índice de utilidad de carne y MAU\% para guanacos adultos (izq.) y subadultos (der.).

Figure 4. Scatter plots of the Meat Utility Index and MAU\% for adult guanacos (left) and subadults (right).

de los elementos meteorizados (Figura 5, C1), los restos con mayores alteraciones $(\geq 4, N=10)$ están relacionados a las áreas de combustión identificadas en las cuadricula XIV-C y XV-C (UE's 34, 59, 60 y 61), aunque también se encuentran especímenes en el sector Este (XVI-C, N=4).

A la vez, cuando se seleccionaron los fragmentos con alteraciones térmicas en donde al menos una porción del hueso presentó carbonización o calcinación ( $\mathrm{N}=80$, Figura $5 \mathrm{C2}$ ), los restos faunísticos mostraron un patrón distribucional semejante. Como se puede apreciar en la Figura 5 C2, las mayores concentraciones aparecen en la cuadricula XIV-C, que a la vez posee la mayor cantidad de fogones identificados ( $\mathrm{N}=6)$. Asimismo, es posible notar una concentración de especímenes que demostraron un proceso de oxidación incompleta en el sector Este de la excavación (XVI-C, $\mathrm{N}=28$ ).

En cuanto a la Figura $5 \mathrm{C}$, la representación exhibe los lugares en donde se concentraron mayor cantidad de elementos en que se pudo identificar marcas de cortes $(\mathrm{N}=14)$, raspados $(\mathrm{N}=4)$ e/o impactos $(\mathrm{N}=77)$. De esta manera se resalta que otros sectores también presentaron las modificaciones pero en menor escala y de manera

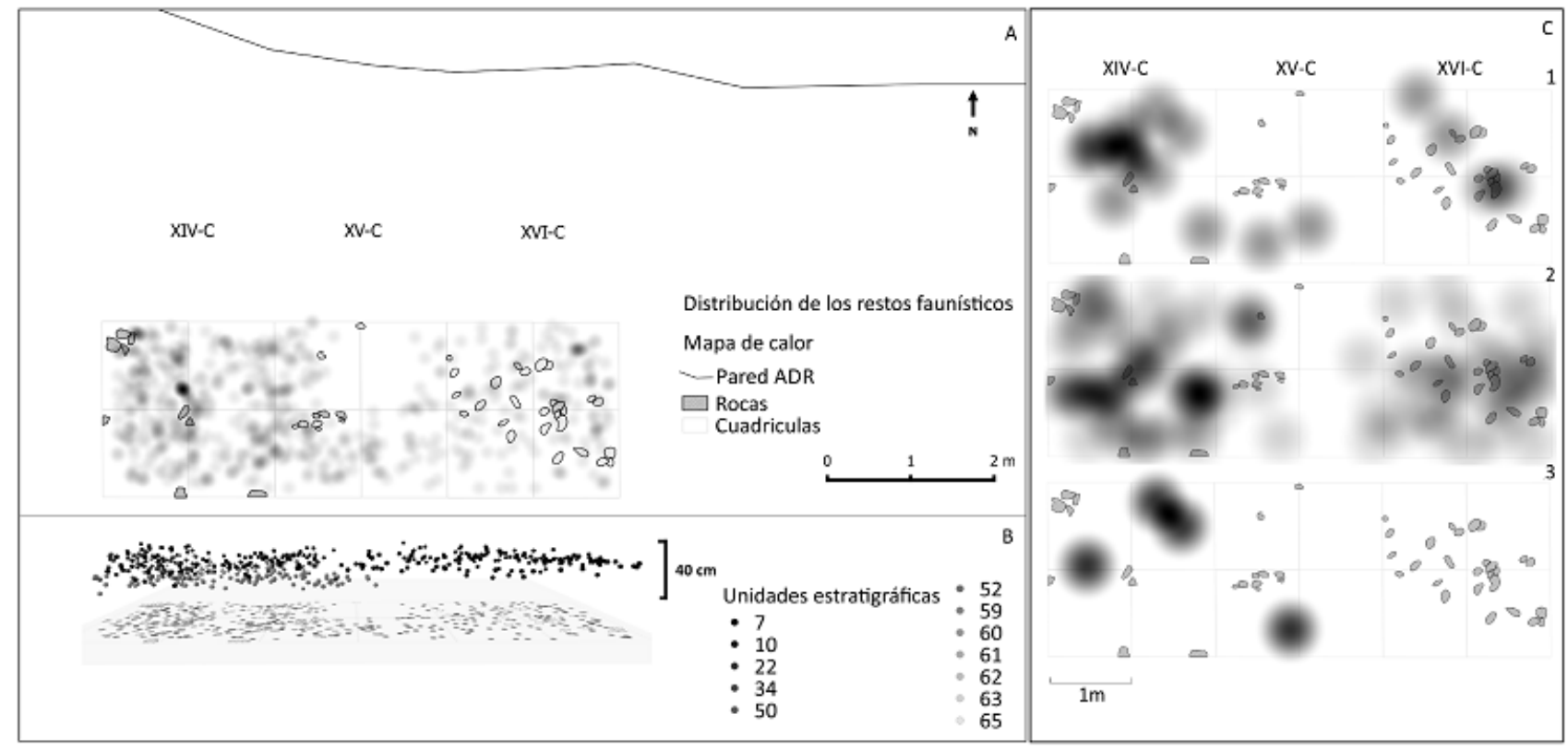

Figura 5. Distribución de los restos faunísticos recuperados. A. Mapa de calor representando la distribución general de los especímenes óseos. B. Representación de la distribución horizontal de los restos faunísticos según su asignación estratigráfica. C. Detalles de la distribución ósea según: meteorización elevada (1); proceso de oxidación térmica incompleta (2); huellas antrópicas de procesamiento (3).

Figure 5. Distribution of the recovered faunal remains. A. Heat map representing the general distribution of bone specimens. B. Representation of the horizontal distribution of the faunal remains by stratigraphic unit. C. Details of bone distribution according to: high weathering (1); incomplete thermal oxidation process (2); anthropic processing traces (3). 
disociada. Asimismo, es importante destacar que la mayor parte de los elementos con alteraciones antrópicas se encuentran en la matriz estratigráfica del bloque temporal (UE7, $\mathrm{N}=50$ ).

En cuanto al registro antracológico recuperado en los sectores aquí tratados, Robledo (2016) afirma que la elección de las leñas para las estructuras de combustión está compuesta por una variedad de especies chaqueñas. Asimismo, el autor propuso que las personas que utilizaron el sitio, eligieron fundamentalmente ramas medianas a chicas, características de fogones con un alto poder calorífico y un rápido consumo (Robledo 2016:120). Además, los fogones del periodo se caracterizaban por presentar grandes cantidades de gasterópodos asociados a estas estructuras (Izeta et al. 2014, Robledo 2016). Por su parte, es interesante destacar, que algunas herramientas líticas como los cortantes y las raederas, que podrían asociarse a un procesamiento relacionado al consumo, se encontraban asociadas a las unidades (UE's 10, 45, 60; véase Tabla 4.3.1 en Caminoa 2016).

\section{Comentarios finales}

De un modo general, el registro faunístico analizado a lo largo del trabajo apunta a que los cazadores-recolectores que ocuparon el Sector B del sitio ADR durante ca. 2970AP se relacionaron ${ }^{1}$ mayoritariamente con los grandes ungulados, representados en la región por los guanacos. En este sentido, y basándonos en los métodos desarrollados a lo largo del trabajo, parecería interesante cuestionar acerca de las posibles razones para la mayor presencia de los guanacos, la baja frecuencia de otros artiodáctilos y, finalmente, el porqué de ciertas ausencias. Con respecto a los guanacos, los estudios del comportamiento de estos camélidos han demostrado que poseen una marcada territorialidad y hasta sedentarismo en los grupos familiares actuales (véase Barri y Fernández 2011, Kaufmann 2009, entre otros), aunque se reconozca que la conducta social de los guanacos puede ser influida por variables ecológicas (Barri y Fernández 2011, Vilá 2012). Estas características deberían favorecer la interacción continuada de los guanacos con los grupos humanos, condición que podría llevar a un proceso de habituación a la presencia humana (ver la propuesta de Yacobaccio y Vilá 2013). En este sentido, para el contexto de Ongamira, pareciera que esta sociabilización ${ }^{2}$ generada a través de la predictibilidad, por parte de los cazadores humanos, acerca de los lugares donde encontrar los grupos de camélidos, fortalecería las relaciones entre los humanos y estos animales cazados (como demuestra el registro faunístico, producto del desecho de esta relación de caza).

\footnotetext{
1 Se utiliza la palabra relacionar bajo el objetivo de abstraerse de una visión económicamente sesgada en donde los animales son vistos únicamente como recursos alimentarios.

2 Partiendo del supuesto que los grupos cazadores-recolectores sociabilizan con el mundo natural de la misma manera que lo hacen entre ellos mismos (véase Fausto 2007; Hill 2011).
}

Por otro lado, los análisis de distribución espacial (Figura 5) sugieren que se llevaban a cabo actividades de combustión con corta duración, según lo inferido a través del registro antracológico (Robledo 2016), y una reiterada utilización del sitio. Acorde a lo anterior, las alteraciones antrópicas manifestadas en los restos óseos (principalmente de guanacos adultos y subadultos, véase Figura 3), permite plantear que se llevaron a cabo actividades de consumo de los animales y procesamiento con diferentes fines.

Con respecto a las herramientas líticas, Caminoa (2016) pudo interpretar la ocurrencia de diversos tipos de cadenas operativas y secuencias completas de producción (principalmente sobre cuarzo), que lo han llevado a considerar que el sitio fue utilizado, por algunos momentos, como un taller para la producción de artefactos. En este sentido, el autor propone que los instrumentos líticos que necesitaban una mayor inversión de trabajo no habrían sido descartados en el sector B del sitio ADR (ibíd. 2016:85). Tal propuesta podría ser ampliada con respecto al conjunto faunístico, si consideramos la cantidad de herramientas líticas relacionadas a la formatización de instrumentos en materias primas blandas (como huesos y maderas) y los escasos elementos óseos formatizados recuperados en el sector B de ADR.

Sin embargo, cabe destacar que González (1952:116), menciona diversos instrumentos elaborados en materia prima ósea en los "pisos superior" y "más profundo" de Ongamira. Con relación al segundo caso, que podría corresponderse al periodo aquí tratado, el autor indica la presencia de tubos en hueso, puntas embotantes en falanges de guanaco, adornos y perforadores (ibíd. 1952). Por esta razón, sería interesante ampliar las excavaciones para poder comprender de manera más ajustada las prácticas que involucraban las personas a los animales procesados en Ongamira.

Con respecto a la baja presencia de cérvidos (véase Tabla 2), la misma podría estar asociada a restricciones en cuanto a su consumo ya que diversos autores han mencionado tabúes asociados a estos ungulados (véase Arenas 2003 para un caso chaqueño). Asimismo, se debería considerar los hábitos solitarios de una de las especies (Mazama guazoubira) como un factor que podría estar relacionado a su escasa presencia en el registro. Por otro lado,es interesante destacar la ausencia del pecarí (Pecari tajacu). Actualmente presente en la región, la especie ha sido registrada apenas en sitios tardíos, asociados a ocupaciones agroalfareras (véase Rivero et al. 2010).

De acuerdo a lo anterior, durante el periodo agroalfarero regional (aprox. 1000 AP), los cambios climáticos inferidos a través de los diferentes proxies (Carignano 1999; Silva et al. 2011; Yanes et al. 2014), que debieron haberse iniciado alrededor de los 3500 AP, proporcionaron 
condiciones climáticas más húmedas que permitieron el avance del Bosque Chaqueño en el Norte de la provincia de Córdoba (Carignano 1999; Medina y Rivero 2007). Estas nuevas condiciones climáticas podrían haber contribuido para la expansión de otras especies (como los ciervos y pecaríes) en la región.

Cabe destacar que Medina y Rivero (2007) han sugerido que la "sobreexplotación" pudo haber diezmado las poblaciones chaqueñas de guanacos a finales del Holoceno tardío. De esta manera, los autores proponen para la región que el aumento en la frecuencia de los cérvidos y especies de menor tamaño estaría relacionado a un proceso de intensificación en las actividades económicas de los grupos humanos, que tendría su fase inicial durante el Holoceno medio (Medina y Rivero 2007; Rivero et al. 2010; Rivero y Medina 2013).

En lo que se refiere a nuestro caso de estudio, para el Holoceno medio se ha planteado que la mayor parte de los animales con menor tamaño corporal han ingresado de manera natural al registro arqueológico (Costa 2015, 2016; Mignino 2016). Sin embargo, existen excepciones como los gasterópodos, dasipódidos y las cáscaras de huevos de reídos, aunque la presencia de estas especies sea ínfima en relación a la de los guanacos (Tabla 2 o Costa 2015, 2016 y Izeta et al. 2014 para un análisis más detallado). En este sentido, queda profundizar los estudios a nivel local y a través de nuevas aproximaciones (p.e. experimentales o de nivel microscópico, entre otras) para inferir de manera más fina acerca de los posibles cambios en las prácticas económicas ocurridas en Ongamira.

Finalmente, la sumatoria en las aproximaciones metodológicas utilizadas en los análisis del registro faunístico de ADR, que han sido en parte presentadas aquí, surge desde un acercamiento que busca la unión de aspectos analíticos e interpretativos y que considera las relaciones económicas de los grupos en cuestión desde una perspectiva más amplia, y quizás menos etnocéntrica (Overton y Hamilakis 2013). En este sentido, creemos que la validez de este tipo de abordaje se justifica a la medida que permite complejizarla discusión acerca de la prácticas económicas de estos grupos humanos, más allá de la búsqueda por calorías.

Córdoba, 31 de agosto de 2016

\section{Agradecimientos}

Esta investigación fue posible gracias al apoyo financiero de los siguientes proyectos: PIP 2014-2016 11220130100137 CO. Arqueología en el Valle de Ongamira, Deptos. Ischilín y Totoral, Córdoba, Argentina, PICT 2011-2122 y SECyT UNC PID 20162017 "Estudios arqueológicos interdisciplinarios de sociedades cazadoras-recolectoras en el valle de Ongamira, Córdoba, Argentina". En este sentido, se agradece a los directores del proyecto Andrés Izeta y Roxana Cattáneo. Finalmente, querría agradecer al equipo del proyecto arqueológico Ongamira: Andrés Robledo, José Caminoa y Gisela Sario. A los ayudantes alumnos: Julián Mignino, Ornella Brancolini, Camila Brizuela, Paula Weihmuller, Isabel Prado, Bernarda Conte y Maxi Córdoba.
Finalmente agradezco a los evaluadores por sus importantes sugerencias para la mejoría del manuscrito. El autor se hace responsable por cualquier error u omisión.

\section{Referencias bibliográficas}

Abe, Y., Marean, C.W., Nilssen, P., Assefa, Z.., \& Stone, E.. (2002). The analysis of cutmarks from archaeological sites: a review and a new GIS image-analysis approach. American Antiquity 66(3), 333-348.

Aguilar, J. P., García Llorca, J., \& Bárcena, J. R. (2011). Estimación de MNE de Bos taurus a partir de imágenes generadas por GIS. En Libro de Actas del IV Congreso Argentino de Arqueometría. Luján.

Arenas, P. (2003). Etnografía y alimentación entre los tobañachilamole\# ek y wichi-Ihuku'tas del Chaco Central (Argentina). $1^{\text {a }}$ Ed. (562 pp). Argentina

Barri, F. R., \& Fernández, M. (2011). Foraging and vigilance time allocation in a guanaco (Lama guanicoe) population reintroduced in Quebrada del Condorito National Park (Córdoba, Argentina). Acta ethologica, 14(2), 103-107.

Binford L.R. (1978). Nunamiut Ethnoarchaeology. New York: Academic Press.

Borrero, L. A. (1990a). Fuego-patagonian bone assemblages and the problem of communal guanaco hunting. En L. B. Davis \& B. O. K. Reeves (Eds.), Hunters of the Recent Past (pp. 373-399). London: Unwin Hyman.

Borrero, L. A. (1990b). Taphonomy of guanaco bones in Tierra del Fuego. Quaternary Research, 34(3), 361-371.

Caminoa, J. M. (2016). Un estudio de tecnología lítica desde la antropología de las técnicas: el caso del Alero Deodoro Roca ca. 2970 AP. Ongamira, Ischilín, Córdoba. South American Archaeology Series 26 (A. D. Izeta, Ed.) Oxford: Archaeopress.

Carignano C. (1999). Late Pleistocene to recent climate change in Córdoba Province, Argentina: Geomorphological evidence. Quaternary International 57-58, 117-134.

Cattáneo, G. R., Di Lello, C., \& Gómez, J. C. (2013). Cuantificación y análisis de la Distribución de rocas útiles para la manufactura de instrumentos a través del uso de sistemas de información geográfica (SIG) en el Área de Piedra Museo, Santa Cruz, Argentina. En M. J. Figuerero Torres \& A. D. Izeta (Eds.), El uso de Sistemas de Información Geográfica en Arqueología Sudamericana (pp. 43-60). South American Archaeology Series 18. Oxford: Archaeopress.

Cattáneo, G.R., Izeta, A.D., \& Takigami, M. (2013b). Primeros fechados radiocarbónicos para el Sector B del sitio Alero Deodoro Roca (Ongamira, Córdoba, Argentina). Revista Relaciones de la Sociedad Argentina de Antropología, 38 (2), 559-567.

Cattáneo, G.R., Izeta, A.D., \& Costa, T. (2015). El patrimonio arqueológico de los espacios rurales de la provincia de Córdoba. 1 a ed. - Córdoba: Universidad Nacional de Córdoba.

Cattáneo, G.R., \& Izeta, A.D. (Ed) (2016). Arqueología en el Valle de Ongamira, $201020151^{\text {a }}$ ed. Córdoba: Universidad Nacional de Córdoba. 
Cebollada Pütz, C., Basso, M., Ruiz de los Llanos, E., \& Kufner, M. (2012). La fauna chaqueña de Córdoba (Argentina) afectada por la transformación agrícola. Ecología Aplicada, 11(2), 77-87.

Costa, T. (2015). Los Humanos, los animales y el territorio. Sus interacciones en el pasado en la Sierras Pampeanas Australes, provincia de Córdoba, Argentina. Facultad de Filosofía y Humanidades, Universidad Nacional de Córdoba, Argentina. Tesis para optar al grado de Doctor en Cs. Antropológicas, 715 pp., 2015.

Costa, T. (2016). Zooarqueología en el Alero Deodoro Roca (ADR). Las interacciones entre personas y animales durante el Holoceno Tardío (1900-3600 AP). En G. R. Cattáneo \& A. D. Izeta (Eds.), Arqueología en el Valle de Ongamira, 2010-2015. Córdoba: Universidad Nacional de Córdoba.

Fausto, C. (2007). Feasting on people. Current Anthropology, $48(4), 497-530$.

Figuerero Torres, M. J., \& Izeta, A. D. (Eds.). (2013). El uso de Sistemas de Información Geográfica (SIG) en arqueología sudamericana: una introducción. B.A.R. International Series S2497. Oxford: Archaeopress.

García Sanjuán, L. (2003). La prospección Arqueológica de superficie y los SIG. Documento de internet disponible en: http://grupo.us.es/atlas/documentos/articulos/ponencia_ cordoba_2003/ponencia_cordoba_2003.pdf. 2003

Geisa, M. (2012). “Determinación de la dieta de guanaco (Lama guanicoe) en estación seca y húmeda de la zona centro del Corredor Biogeográfico del Chaco Árido, Córdoba".Universidad Nacional de Córdoba, Facultad de Ciencias exactas, físicas y naturales, Córdoba, Argentina. Tesis de licenciatura no publicada, 82 pp. 2012.

Gianotti, C., \& Bonomo, M. (2013). De montículos a paisajes: procesos de transformación y construcción de paisajes en el sur de la Cuenca del Plata. Comechingonia. Revista de Arqueología, 17(2), 59-94.

González A.R. (1943). Restos arqueológicos del Abrigo de Ongamira. I Congreso de Historia Argentina del Norte y Centro, Tomo I, 143-157.

González A. R. (1952). Antiguo horizonte precerámico en las Sierras Centrales de la Argentina. Runa, V, 110-133.

Gordillo, S., Izeta, A. D., Costa, T., Boretto. G., \& Cattáneo, R. (2013). El borus enano de Ongamira: una especie endémica del noroeste de la Provincia de Córdoba en contexto arqueológico de cazadores recolectores. En Hammond y Zubimendi (Eds.), Arqueología y malacología: abordajes metodológicos y casos de estudio en el Cono Sur. Buenos Aires: Fundación de Historia Natural Félix de Azara.

Grayson, D.K. (1984). Quantitative Zooarchaeology. New York: Academic Press.

Grosjean, M., Núñez, L., Cartajena, I. \& Messerli, B. (1997). MidHolocene climate and culture change in the Atacama Desert, northern Chile. Quaternary Research, 48(2), 239-246.

Gutiérrez, M. A. (2009). Tafonomía: ¿Tiranía o Multivocalidad? En R. Barberena, K. Borazzo \& L.A. Borrero (Eds.), Perspectivas
Actuales en Arqueología Argentina, pp. 55-87. 1a ed. - Buenos Aires: IMHICIHU.

Hegmon, M. (2003). Setting theoretical egos aside: Issues and theory in North American archaeology. American Antiquity, $68(2), 213-243$.

Hill, E. (2011). Animals as agents: Hunting ritual and relational ontologies in Prehistoric Alaska and Chukotka. Cambridge Archaeological Journal, 21(3), 407-426.

Izeta, A. D. (2007). Zooarqueología del sur de los valles Calchaquies (Provincias de Catamarca y Tucumán, República Argentina): Análisis de conjuntos faunísticos del primer milenio A.D. B.A.R. International Series S1612. Oxford: John and Erica Hedges.

Izeta, A. D. (2013). Perfil de fragmentación ósea: una aproximación basada en el análisis de imágenes generadas por sistemas de información geográfica (SIG). En M. J. Figuerero Torres \& A. D. Izeta (Eds.), El uso de Sistemas de Información Geográfica en Arqueología Sudamericana (pp. 173-184). South American Archaeology Series 18. Oxford: Archaeopress.

Izeta, A. D., Costa, T., Gordillo, S., Cattáneo, R., Boretto, G., \& Robledo, A. (2014). Los gasterópodos del sitio Alero Deodoro Roca, Valle de Ongamira (Córdoba, Argentina). Un análisis preliminar. Revista Chilena de Antropología, 29 (1), 74-80.

Kaufmann, C. (2009). Metodologías para la construcción de perfiles de mortalidad y determinación de la estacionalidad en restos óseos de Guanaco (Lama guanicoe): su aplicación a sitios arqueológicos de la región pampeana. Tesis de Doctorado. FCNyM, UNLP. MS

Leynaud, G. C. \& Bucher, E. H. (2005). Restoration of Degraded Chaco woodlands: effects on reptile assemblages. Forest ecology and Management, 213(1), 384-390.

López M, P., Cartajena F, I., Santander, B., Villalón, D., Sáez, A., \& Rivera, B. (2016). Procesamiento de guanacos durante el Arcaico Tardío del Norte Semiárido de Chile: un acercamiento zooarqueológico y espacial intrasitio. Chungará (Arica), 48(2), 243-258.

Lyman, R. L. (1994). Vertebrate Taphonomy. Cambridge Manuals in Archaeology, Cambridge University Press.

Magnin, L. A. (2013). Incorporación de Sistemas de InformaciónGeográfica a Estudios Arqueológicos de Cazadores Recolectores, Sector Norte del Macizo Central de Santa Cruz, Argentina. En M. J. Figuerero Torres \& A. D. Izeta (Eds.), El uso de Sistemas de Información Geográfica en Arqueología Sudamericana (pp. 9-30). South American Archaeology Series 18. Oxford: Archaeopress.

Maguire, D., Goodchild, M. \& Rhind, D. (1991). Geographical information system: principles and applications, Longman Scientific technical, Harlow, Essex, Wiley, New York.

Mainland, I., Card, N., Saunders, M. K., Webster, C., Isaksen, L., Downes, J., \& Littlewood, M. (2014). 'SmartFauna': a microscale GIS-based multi-dimensional approach to faunal deposition at the Ness of Brodgar, Orkney. Journal of Archaeological Science, 41, 868-878. http://doi.org/http://dx.doi.org/10.1016/j. jas.2013.10.019 
Marciniak, A. (2001). Scientific and Interpretative components in social zooarchaeology. The case of early farming communities in Kujavia. Archaeologia Polonia, 39, 87-110.

Marean, C.W., Y. Abe, P. Nilssen, \& Stone, E. (2001). Estimating the Minimum Number of Skeletal Elements (MNE) in Zooarchaeology: a Review and a New Image-analysis GIS Approach. American Antiquity, 66(2), 333-348.

Marín Arroyo, A. B. (2008). El yacimiento paleolítico de la Cueva del Mirón: Resultados de la aplicación de nuevas metodologías arqueozoológicas. En Zooarqueología hoy: encuentros hispanoargentinos (pp. 69-88). Burgos: Servicio de Publicaciones.

Matteucci, S. D. \& Scheinsohn, V. (2004). Procesamiento de imágenes, SIG y modelos ecológicos aplicados a la arqueología. GeoFocus (Artículos), 4, 93-109.

Medina, M.E., \& Rivero, D. (2007). Zooarqueología, Lama guanicoe y dinámica evolutiva del Chaco Serrano. Mundo de Antes, 5, 211-234.

Menghin, O. \& González, A. R. (1954). Excavaciones arqueológicas en el yacimiento de Ongamira, Córdoba (Rep. Argentina). Nota preliminar. Antropología. Notas del Museo de La Plata, XVII (67), 213-274.

Mengoni Goñalons, G. L. (1999). Cazadores de Guanacos de la estepa patagónica. Buenos Aires: Sociedad Argentina de Antropología, Colección Tesis Doctorales.

Mignino, J., Izeta, A. D., \& Martínez, J. J. (2016). Micromamíferos del Holoceno Tardío del Valle de Ongamira: Taxonomía, tafonomía y reconstrucción paleoambiental En: Cattáneo, R. \& Izeta A. (Ed). Arqueología en el Valle de Ongamira, Córdoba (2010-2015). IDACOR-Museo de Antropología, UNC, Argentina.

Montes, A. (1943). Yacimiento Arqueológico de Ongamira. I Congreso de Historia del Norte y Centro, tomo I, 239-252. Córdoba.

Moreno, E. (2013). Estrategias de caza y paisajes culturales en Antofagasta de la Sierra, Catamarca. Comechingonia, 17(2), 165-190.

Nicholson, R. (1993). A morphological investigation of burnt animal bone and an evaluation of its utility in archaeology. Journal of Archaeological Science, 20(4), 411-428.

Orton, D. C. (2012). Taphonomy and interpretation: An analytical framework for social zooarchaeology. International Journal of Osteoarchaeology, 22(3), 320-337.

Overton, N. J., \& Hamilakis Y. (2013). A manifesto for a social zooarchaeology. Swans and other beings in the Mesolithic. Archaeological Dialogues, 20(02), 111-136.

Pascual, R. (1954) Restos de Vertebrados Hallados en el Abrigo de Ongamira (Córdoba). Notas del Museo de La Plata, Antropología, XVII (67), 269-274.

Pastor, S., Murrieta Flores, P., \& García Sanjuán, L. (2013). Los SIG en la arqueología de habla hispana: Temas, técnicas y perspectivas. Comechingonia, 17(2), 9-29.

Pastore, F., \& Methol, E. (1953). Descripción geológica de la Hoja 19i, Capilla del Monte (Córdoba). Carta geológico-económica de la República Argentina. Escala 1:200.000. Buenos Aires. Ministerio de Industria y Comercio de la Nación.

Ringuelet, R. A. (1961). Rasgos fundamentales de la zoogeografía de la Argentina. Physis, 22(63), 151-170.

Rivero, D., Medina, M. E., Recalde, A., \& Pastor, S. (2010). Variabilidad en la Explotación de Recursos Faunísticos durante el Holoceno en las Sierras de Córdoba (Argentina): Una aproximación Zooarqueológica. En Zooarqueología a principios del siglo XXI: Aportes Teóricos, Metodológicos y Casos de Estudio. I Congreso Nacional de Zooarqueología Argentina. 321 - 331. Buenos Aires.

Rivero, D. \& Medina, M. E. (2013). Human Colonization, Diet Breadth and Niche Construction during Sierras of Córdoba Holocene (Argentina). En: H. Muscio, \& M. Cardillo (Ed.). Darwin's Legacy: The State of Evolutionary Archaeology in Argentina (pp 76 - 88). SAMAR Series 24, A. D. Izeta (Ed). Oxford: Archaeopress.

Robledo A.I. (2016). Estudios antracológicos en los espacios de combustión del Alero Deodoro Roca - Ongamira (Córdoba). (166 pp) South American Archaeology Series 25. A. D. Izeta (Ed). Oxford: Archaeopress.

Russell, N. (2012). Social zooarchaeology: Humans and animals in prehistory. (562 pp) Cambridge: Cambridge University Press.

Sario, G, \& Salvatore, M. (2013). Aplicación de un SIG a un caso arqueológico: sitio 3 de la localidad Estancia La Suiza (San Luis, Argentina). GeoFocus (Informes y Aplicaciones), 13(1), 38-47.

Silva L.C.R., M.A. Giorgis, M. Anand et al. (2011). Evidence of shift in C4 species range in central Argentina during the late Holocene. Plant and Soil 349: 261-279.

Todd, L. C. (1987). Taphonomy of the Horner II Bonebed. En G.C. Frison y L.C. Todd (Eds.). The Horner Site: The Type Site of the Cody Cultural Complex. Pp. 107-198. Orlando: Academic Press.

Vilá, B. (2012). Camélidos sudamericanos. Colección ciencia joven 40. Buenos Aires: Eudeba.

Wheatley, D.W., \& Gillings, M. (2002). Spatial Technology and Archaeology. The Archaeological Application of GIS. Londres, Taylor and Francis.

Yacobaccio, H. D, \& Vilá, B. (2013). La domesticación de los camélidos andinos como proceso de interacción humana y animal. Intersecciones en antropología, 14(1), 227-238.

Yanes Y., Izeta, A.D., Cattáneo, G. R., Costa, T., \& Gordillo, S. (2014). Holocene paleoenvironmental ( 4.5-1.7 cal. kyr BP) conditions in central Argentina inferred from entire-shell and intra-shell stable isotope composition of land snails. The Holocene 24 (10): 1193-1205.

Zak M.R., Cabido, M., \& Hodgson, J. G. (2004). Do subtropical seasonal forests in the Gran Chaco, Argentina, have a future? Biological Conservation 120, 589-598

Zak M.R., Cabido, M., Cáceres, D., \& Díaz, S. (2008). What Drives Accelerated Land Cover Change in Central Argentina? Synergistic Consequences of Climatic, Socioeconomic, and Technological Factors. Environmental Management, 42, 181-189. 1 